\title{
Comparison of imaging techniques in the diagnosis of bridging bronchus
}

\author{
W. Baden*, J. Schaefer* , M. Kumpf*, N. Tzaribachev*, T. Pantalitschka*, \\ A. Koitschev ${ }^{\top}$, G. Ziemer ${ }^{+}$, J. Fuchs ${ }^{\S}$ and M. Hofbeck*
}

ABSTRACT: Bridging bronchus (BB) is a rare, congenital bronchial anomaly that is frequently associated with congenital cardiac malformations, especially left pulmonary artery sling. It represents an anomalous bronchus to the right originating from the left main bronchus. Discrimination from other bronchial anomalies is important, since BB is frequently associated with bronchial stenoses due to abnormal cartilage rings.

This case study describes the findings of bronchoscopy, bronchography and multidetector computed tomography (MDCT) in three patients. Bronchoscopy was helpful in the description of the severity and length of bronchial stenoses. However, it was not possible to establish a diagnosis of BB based on this method in two patients, since it is difficult or even impossible to differentiate the bifurcation from the pseudocarina. It was not possible to establish the correct diagnosis in all patients based on bronchography or MDCT. MDCT was able to depict the relationship of bronchial and vascular structures, which is particularly important in patients with pulmonary artery sling.

Multidetector computed tomography is preferable to bronchography as it is less invasive and due to its short acquisition time it can be performed in children with severe respiratory disease. In the current authors' experience, detection of cartilage rings still requires flexible bronchoscopy.

KEYWORDS: Bridging bronchus, bronchial arborisation disorder, congenital heart disease, pulmonary sling, supernumerary bronchus

\footnotetext{
A nomalies of tracheal and bronchial branching have been reported in $0.1-$ $1.9 \%$ of children [1]. The spectrum of these anomalies comprises displaced, supernumerary and bridging bronchus (BB), as well as oesophageal origin of a main stem or accessory bronchus (table 1). The BB represents an aberrant bronchus that originates from the left main stem bronchus, crossing ("bridging") the mediastinum to supply the right medium and/or lower lobes. It originates from a pseudocarina at a lower level than the normal right main stem bronchus which arises at the level of a normal bifurcation. However, the right main stem bronchus, may be absent or present only as a short tracheal diverticulum. In previous reports the diagnosis has frequently been delayed until autopsy. The present study describes the findings of multidetector computed tomography (MDCT) in comparison with bronchoscopy and bronchography in three patients with BB.
}

\section{PATIENTS AND METHODS}

Between March 1997 and March 2005, three children with BB were diagnosed in the authors' tertiary referral centre. During the diagnostic work-up, all patients underwent at least one bronchoscopy and MDCT of the thorax; two out of three patients had additional bronchography.

Tracheobronchoscopy was performed under general anaesthesia with flexible endoscopes (Pentax $3.5 \mathrm{~mm}$ (Pentax Europe GmbH, Munich, Germany) and Storz $2.5 \mathrm{~mm}$ (Karl Storz GmbH \& Co. KG, Tuttlingen, Germany) outer diameter). Bronchography was performed by endotracheal instillation of 1-2 mL contrast medium (iomeprol 300; $30 \mathrm{mg}$ iodine $/ 100 \mathrm{~mL}$ ) diluted $1: 1$ with $0.9 \%$ saline. MDCT scans were performed on a Siemens system (Erlangen, Germany) with four- or 64-row detectors. The tube voltage was $120 \mathrm{kV}$ and tube current was adapted to body weight using a low-dosage technique. Collimation was $4 \times 1 \mathrm{~mm}$ or $64 \times 0.6 \mathrm{~mm}$
AFFILIATIONS

*Depts of Paediatric Cardiology and Pulmology,

"Radiology,

"ENT,

${ }^{+}$Cardiovascular and Thoracic

Surgery, and

§Paediatric Surgery, Children's Hospital, University of Tuebingen, Tuebingen, Germany.

CORRESPONDENCE

W. Baden

Dept of Paediatric Cardiology and Pulmology

Children's Hospital

University of Tuebingen

Hoppe-Seyler-Str. 1

D-72076 Tuebingen

Germany

Fax: 497071295054

E-mail: winfried.baden@med

uni-tuebingen.de

Received:

April 142007

Accepted after revision:

November 142007

STATEMENT OF INTEREST

None declared. 


\begin{tabular}{l} 
TABLE 1 Classification of anomalous bronchus \\
Displaced tracheal bronchus \\
A lobar or segmental bronchus arises in an abnormal position from \\
the trachea or ipsalateral bronchus \\
Bridging bronchus \\
A lobar or segmental bronchus arises from the contralateral bronchus \\
Supernumerary tracheal bronchus \\
An accessory lobar or segmental bronchus arises from the trachea \\
or bronchus \\
Supernumerary oesophageal bronchus \\
An accessory bronchus arises from the oesophagus \\
(broncho-oesophageal fistula) \\
Oesophageal lung \\
A main stem bronchus arises from the oesophagus \\
\hline
\end{tabular}

The classification is based on data from [1-3]

with a slice thickness of $1.25 \mathrm{~mm}$ or $0.6 \mathrm{~mm}$. Rotation time was 0.5 or $0.3 \mathrm{~s}$, with a table speed of $8 \mathrm{~mm} \cdot \mathrm{s}^{-1}$ or $76 \mathrm{~mm} \cdot \mathrm{s}^{-1}$. Depending on the clinical condition of the patient the examinations were performed while the patients were sedated (midazolam, ketamine), under spontaneous breathing or under general anaesthesia. Patients one and three had clinical suspicion of air trapping and peripheral obstruction so a series of two MDCT scans were performed in expiratory and in inspiratory breath-hold to distinguish between bronchomalacia and stenosis. Under sedation this was achieved by a short period of hyperventilation with a breathing bag and a face mask. By means of a positive end-expiratory pressure (PEEP) valve on the breathing bag the MDCT was obtained during the resulting short period of apnoea as inspiratory hold (high PEEP) or expiratory hold (low PEEP). Contrast medium $2 \mathrm{~mL} \cdot \mathrm{kg}^{-1}$ body weight (iomeprol 400 (40 mg of iodine/100 mL), Bracco-Byk Gulden, Konstanz, Germany) was applied through a 22- or 24-gauge needle via an antecubital vein at a rate of 1.2$3 \mathrm{~mL} \cdot \mathrm{s}^{-1}$. All computed tomography (CT) display techniques were interactive without lavish post-processing, resulting in a process time of only a few minutes. Virtual endoscopic views were generated by the use of the surface-shaded display technique. Bronchographic CT views were produced by means of the transfer function for air using the volume rendering technique.

\section{CASE REPORTS}

\section{Case 1}

Patient one was a female born in the 41st gestational week with a birth weight 3,350 g. Neonatal examination revealed anal atresia with rectovaginal fistula, hypoplastic uterus with absent left ovarium and dysplastic os sacrum. Echocardiography and
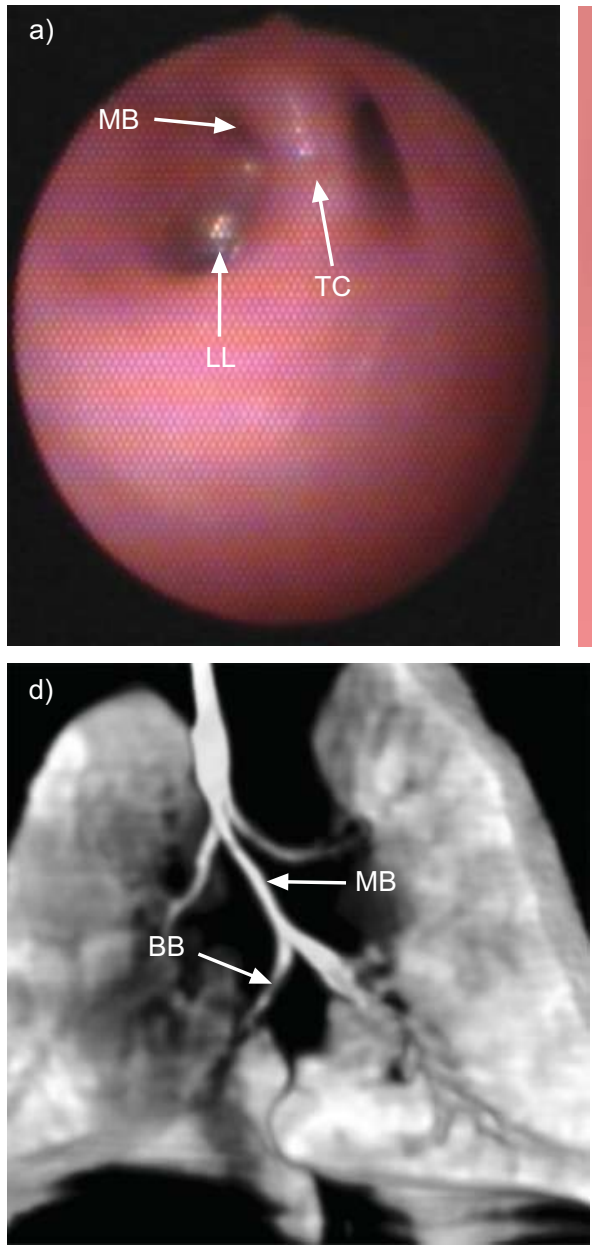
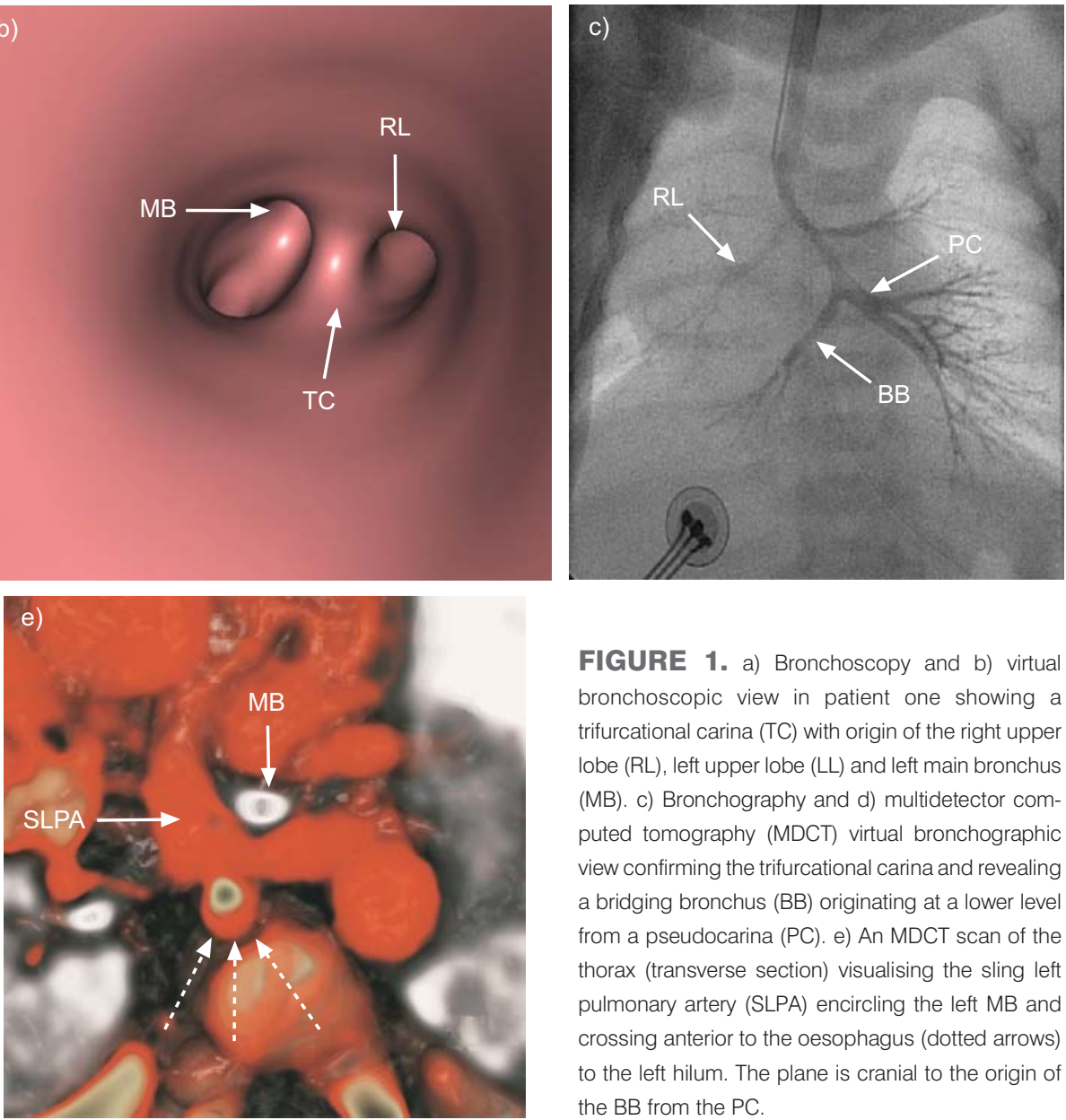

FIGURE 1. a) Bronchoscopy and b) virtual bronchoscopic view in patient one showing a trifurcational carina (TC) with origin of the right upper lobe (RL), left upper lobe (LL) and left main bronchus (MB). c) Bronchography and d) multidetector computed tomography (MDCT) virtual bronchographic view confirming the trifurcational carina and revealing a bridging bronchus (BB) originating at a lower level from a pseudocarina (PC). e) An MDCT scan of the thorax (transverse section) visualising the sling left pulmonary artery (SLPA) encircling the left MB and crossing anterior to the oesophagus (dotted arrows) to the left hilum. The plane is cranial to the origin of the $\mathrm{BB}$ from the $\mathrm{PC}$ 
cardiac catheterisation demonstrated tetralogy of Fallot and left pulmonary artery sling. Chest radiography revealed dextroversio cordis, pulmonary dystelectasis and a shift of the entire mediastinum and trachea to the right suspicious of right lung hypoplasia. Flexible bronchoscopy combined with bronchography showed atypical branching of the right and left upper lobe bronchus at the level of a normal bifurcation and an elongated left main bronchus. The left main bronchus ended in a trifurcation, giving rise to the right lower lobe bronchus (BB) and to bronchi in the lingula and left lower lobe (fig. 1). An MDCT scan of the thorax was performed at 6 days of age in combination with reconstruction of the bronchial tree allowing virtual bronchoscopy. These examinations showed a bifurcation at the level of the fourth and fifth thoracic vertebrae and the BB originating at the level of sixth and seventh thoracic vertebrae. There was neither air trapping nor segmental atelectasis, although the left main bronchus as well as the BB to the right lower lobe appeared narrow. Contrast medium application confirmed sling left pulmonary artery (SLPA; fig. 1) encircling the atypical left main bronchus at the level of the pseudocarina (type IIA according to the classification of WeLLS et al. [4]; fig. 2). The SLPA caused no compression of the airways. Surgical correction of tetralogy of Fallot was performed at 9 days of age. Due to absent airway obstruction the SLPA was left untouched. At 7 yrs of age the patient continues to have an excellent haemodynamic result and as yet has not required surgical intervention for airway obstruction.

\section{Case 2}

A male was born at 42 weeks of gestation with a birth weight of $2,540 \mathrm{~g}$. At 2 days of age, and a systolic murmur was noted and echocardiography revealed a large perimembraneous ventricular septal defect, secundum atrial septal defect and partial anomalous pulmonary venous return of the right upper pulmonary vein. Following surgical correction of the cardiac malformation at the age of 5 months, the child could not be weaned from the respirator. Flexible bronchoscopy at the age of 6 months showed a buttonhole subglottic stenosis. Following tracheostomy at 8 months of age the child remained respirator dependant. An MDCT scan with endoscopic and bronchographic view (fig. 3) reconstruction at 6 yrs of age revealed a tracheal bifurcation at the level of the fifth thoracic vertebrae giving rise to the right upper lobe bronchus and to a stenotic left bronchus. A pseudocarina at the level of the sixth and seventh thoracic vertebrae gave rise to a $\mathrm{BB}$ connecting to the right middle and lower lobe and to the left main bronchus with normal arborisation of the left lung. Repeat bronchoscopy revealed that the severe stenosis of the left main bronchus was caused by two circular cartilage rings (fig. 3). At the age of 6 and 7 yrs the child could be weaned from respirator therapy and cannula, respectively. Despite the stenotic left bronchus there is stridor only on exertion and the patient is developing normally.

\section{Case 3}

A young male was born in the 36th gestational week with a birth weight of $1,980 \mathrm{~g}$. On the first day of life, oesophageal atresia was diagnosed. Echocardiography revealed a large perimembranous ventricular septal defect. As there was a long atretic segment, primary anastomosis of the oesophagus was impossible. The child underwent creation of a gastrostomy and a collar oesophageal stoma. The ventricular septal defect was
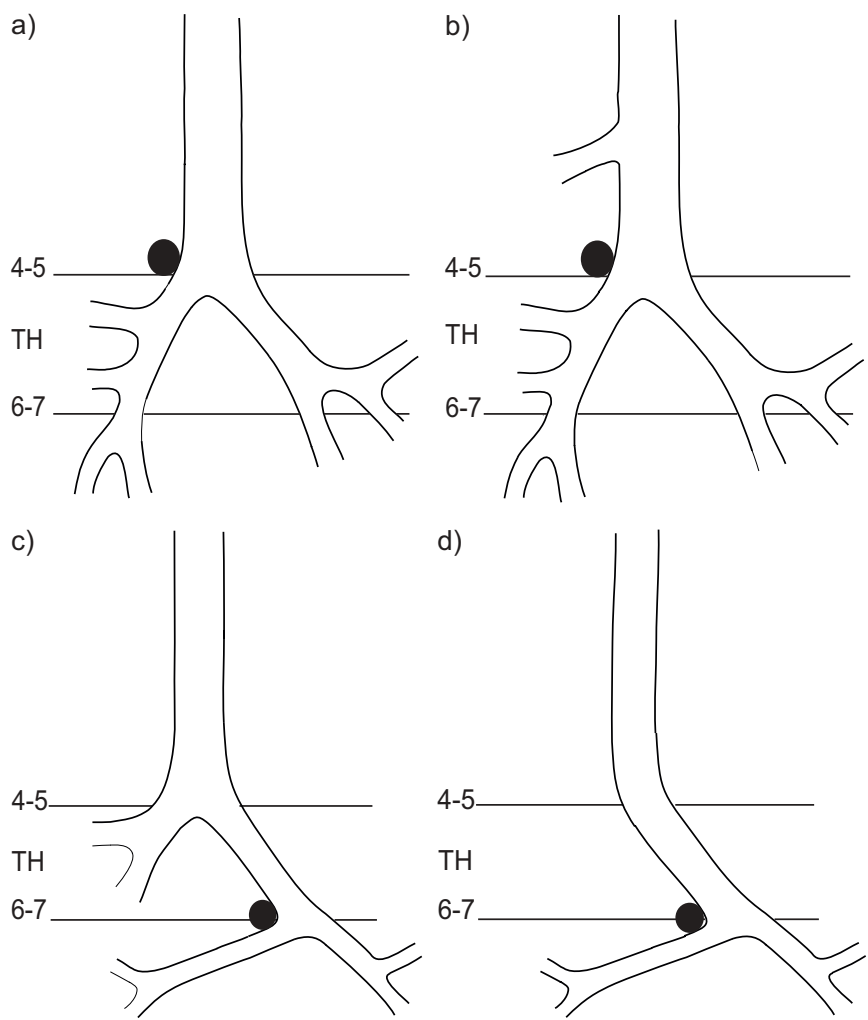

FIGURE 2. Tracheobronchial arborisation disorders associated with sling left pulmonary artery (SLPA; - ). a, b) Type IA and IB SLPA, respectively, and c, d) Type IIA and IIB SLPA, respectively. Type IA refers to SLPA with normal tracheobronchial anatomy and normal bifurcation at the level of fourth and fifth thoracic vertebrae $(\mathrm{TH})$. In type IB (b), there is an additional tracheal bronchus on the right upper lobe. Bridging bronchus (BB) is present in type II of SLPA. In type IIA (c), BB originates from a pseudocarina at the level of sixth and seventh thoracic vertebrae with right upper lobe bronchus originating from the carina at the level of fourth and fifth thoracic vertebrae. In type II B (d), the right upper lobe bronchus is absent. The SLPA crosses over the right bronchus in type I and the BB in type II. The classification of SLPA is according to WELLS et al. [4]

palliated by pulmonary artery banding at the age of 4 weeks. Surgical correction of the oesophageal atresia was performed at the age of 12 months. Pre-operative MDCT of the thorax at the age of 10 months (fig. 4) followed by bronchoscopy revealed a $\mathrm{BB}$ originating from the left main bronchus at the level of the seventh thoracic vertebra supplying the right middle and lower lobe. However, a subglottic tracheooesophageal fistula was missed initially, but detected by repeat bronchoscopy and bronchography performed during spontaneous breathing. The fistula was closed surgically 3 weeks after repair of the oesophageal atresia. Finally, surgical repair of the ventricular septal defect was performed at the age of 13.5 months. At the age of 4 yrs the child was free from respiratory symptoms.

\section{DISCUSSION}

GONZALEZ-CRUSSI et al. [5] introduced the term "bridging bronchus" for an airway malformation in which the right upper lobe is supplied by the right main bronchus while the right middle and lower lobes are supplied by a bronchus that originates from the left main bronchus crossing ("bridging") 

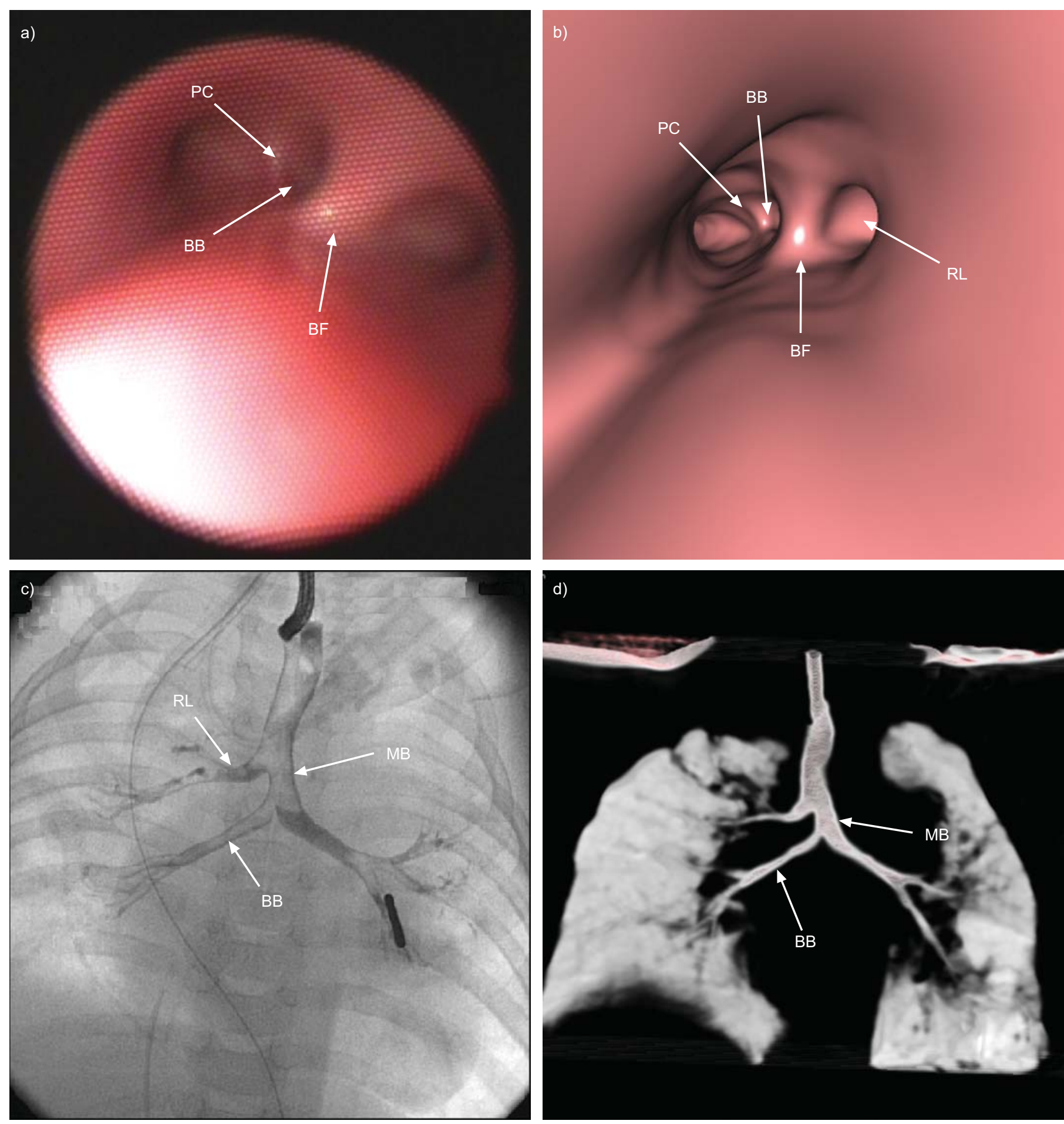

FIGURE 3. a) Bronchoscopy and b) multidetector computed tomography (MDCT) virtual bronchoscopic view from the distal trachea in patient three showing the bifurcation $(\mathrm{BF})$, right upper lobe $(\mathrm{RL})$ and left main bronchus $(\mathrm{MB})$. The pseudocarina $(\mathrm{PC})$ is at the bottom of the left $\mathrm{MB}$ is, with originating bridging bronchus (BB). c) Bronchography and d) MDCT virtual bronchographic view confirm the BB originating after a short distance from the left MB.

the mediastinum from left to right. The BB presents in two anatomic subtypes [4]. The first variant is characterised by a trachea of normal length with a normal number of tracheal rings. It bifurcates at the level of the interspace between the 4 th and 5th thoracic vertebrae into a right main bronchus, exclusively supplying the right upper lobe, and into the left main bronchus (fig. 2). The BB originates from the left main bronchus at the level of the interspace between the sixth and seventh thoracic vertebrae and supplies the right lower and middle lobes. In the second variant of $\mathrm{BB}$ the right main bronchus, originating at the level of the interspace between the fourth and fifth thoracic vertebrae, is only present as a short 

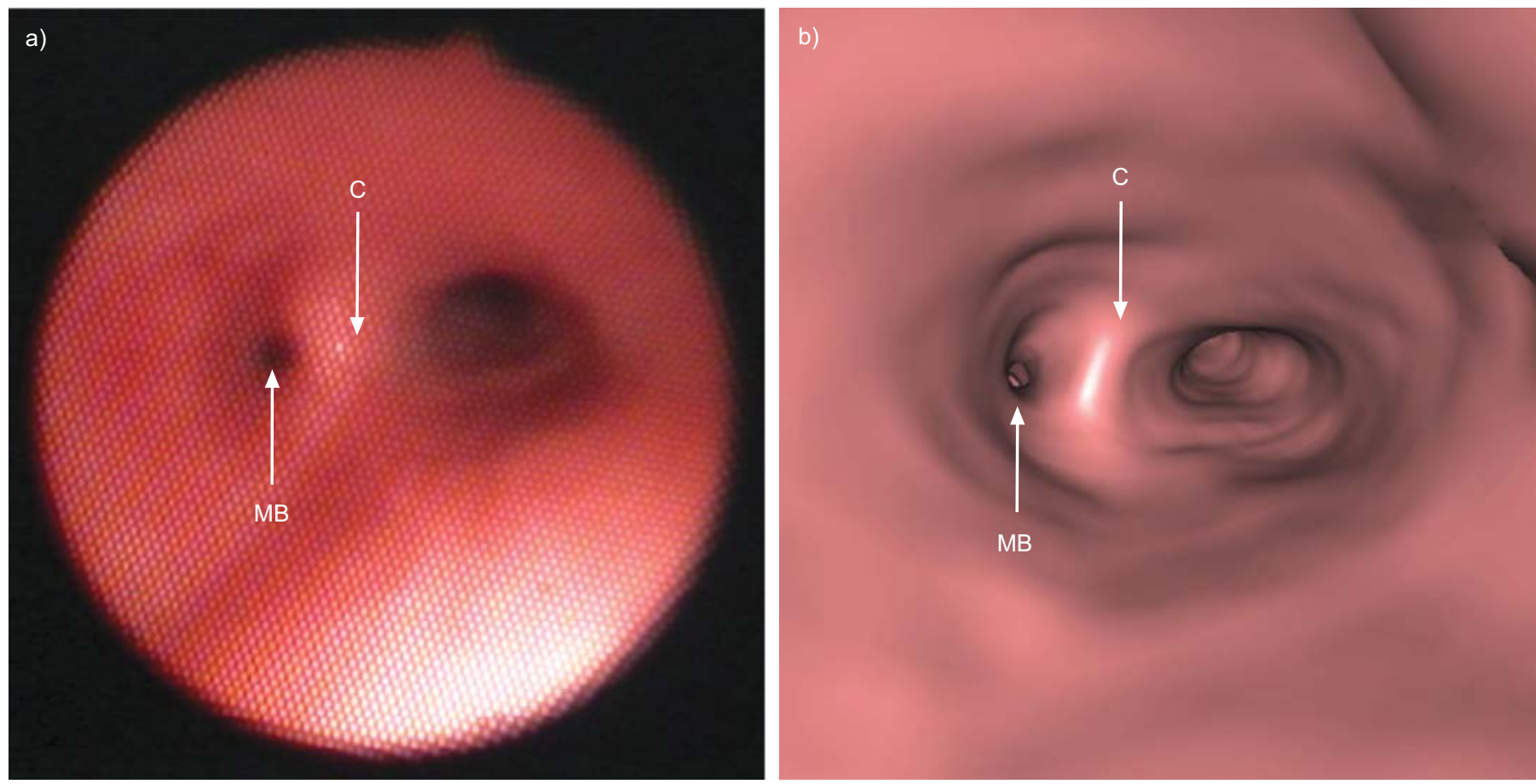

c)

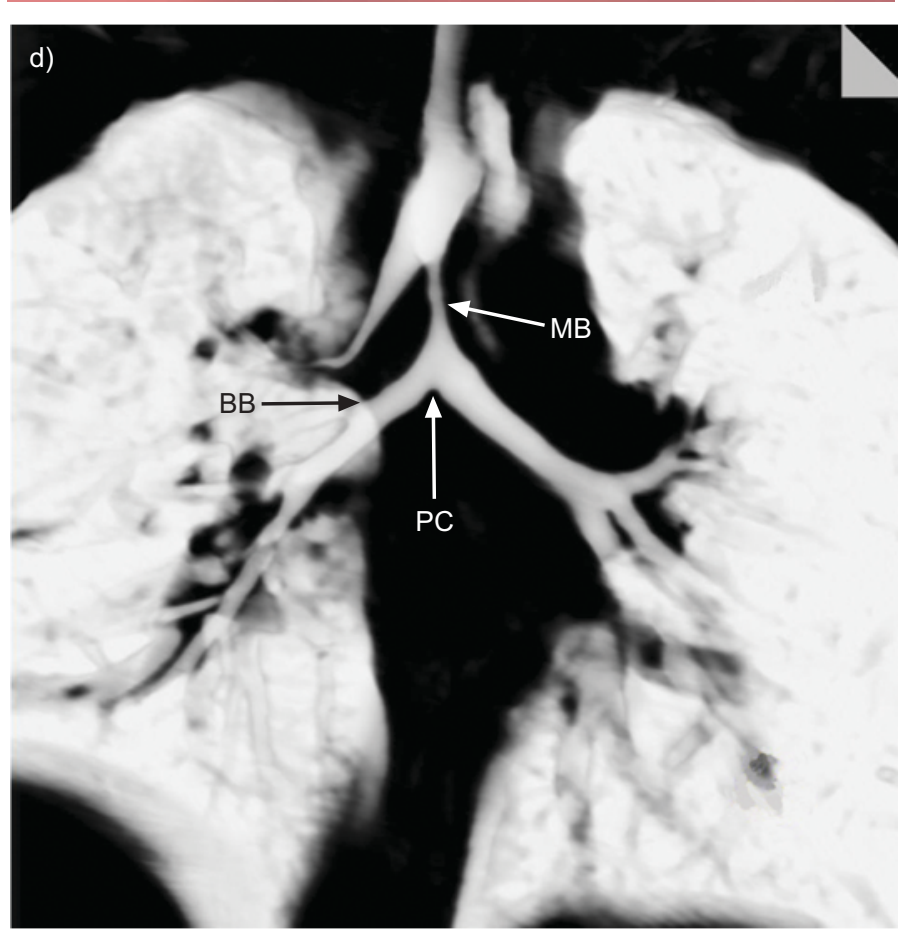

FIGURE 4. a) Bronchoscopy and b) multidetector computed tomography (MDCT) virtual bronchoscopic view in patient two demonstrated a tight stenosis of the left main bronchus (MB) distal to the carina (C). C) MDCT virtual bronchoscopy and d) multidetector computed tomography (MDCT) bronchography show the pseudocarina (PC) bifurcating into the bridging bronchus (BB) and left MB.

tracheal diverticulum or may be even absent. The BB supplying the right middle and lower lobe originates at the level of the interspace between the sixth and seventh thoracic vertebrae. The right lung in patients with this subtype is frequently hypoplastic [4]. In both variants the angle of origin of the BB from the left main bronchus is increased compared with normal bifurcation giving the "pseudocarina" the configuration of an inverted $\mathrm{T}$ [4, 6-8]. All of the studied patients had the first variant of this anomaly.

$\mathrm{BB}$ is the most frequent (78\%) tracheobronchial malformation encountered in children with SLPA. SLPA is an anomaly characterised by an aberrant left pulmonary artery arising from the proximal right pulmonary artery, passing posteriorly 
over the proximal right bronchus and coursing between the trachea and oesophagus to the left hilum [9]. According to WeLLs et al. [4], classification of bronchial anomalies in patients with SLPA discriminates two variants of $\mathrm{BB}$, as mentioned previously, as type IIA and IIB (fig. 2). However, it has been well documented in the literature [10] and now confirmed by two of the case study patients that BB may occur without pulmonary sling.

Recognition of $\mathrm{BB}$ has frequently been delayed due to misinterpretation of the pseudocarina. If the junction of the BB with the left main bronchus is misinterpreted as tracheal bifurcation, the right main bronchus will be classified incorrectly as tracheal bronchus (bronchus suis). Patients with tracheal bronchus can be differentiated by the fact that the origin of the accessory bronchus to the right upper lobe is found at a higher level than a normal bifurcation, which is expected at the level of the interspace between the fourth and fifth thoracic vertebrae. Clinical differentiation is important, since patients with BB frequently show tracheal and left main bronchus stenoses with varying extent of absence of the pars membranacea of the trachea and abnormal tracheal and main bronchial cartilage rings, as in patient two [11]. Furthermore, in a significant number of patients, BB is associated with additional cardiovascular, skeletal, genitourinary and abdominal malformations [11, 12]. Therapy should include the management of cardiac abnormalities, extra cardiac malformations, as well as surgical procedures for relief of bronchial stenoses [13] due to pulmonary sling or cartilage rings.

\section{Imaging procedures in infants with BB}

According to the present authors' experience, diagnosis of $\mathrm{BB}$ still requires the combination of different imaging modalities. Bronchoscopy is valuable in the assessment of tracheal and bronchial stenoses and is presently the method of choice to assess the presence or absence of cartilage rings, which can be detected based on the absence of the dorsal pars membranacea. It is difficult, however, to establish the diagnosis of BB based exclusively on bronchoscopy, since it is almost impossible to differentiate the true bifurcation from the pseudocarina.

In the majority of cases reported in the literature, the clinical diagnosis of BB has been established by bronchography, since this examination allows the recognition of the abnormal level of the pseudocarina, as well as describing the tracheal and bronchial anatomy and bronchial stenoses [14]. It requires less radiation than MDCT of the thorax but it cannot be performed without general anaesthesia in children. Furthermore, due to the requirement of instillation of contrast medium, this method is rather invasive in infants who frequently present in severe respiratory distress.

According to the present authors' experience, MDCT presently appears to be the best primary imaging technique. Threedimensional (3D) reconstruction of the trachea allows recognition of the bifurcation and pseudocarina, and of its spatial relation to other anatomic structures in the thorax (e.g. thoracic vertebrae), as well as recognition of the increased angle (inverted T) of the pseudocarina. The presence of an accompanying SLPA can be depicted unequivocally following intravenous application of contrast medium. A good correlation has been found between bronchial anatomy including severity and length of bronchial stenoses as visualised in bronchography and virtual bronchography based on 3D reconstruction of MDCT. However, the current authors were unable to make statements regarding the presence or absence of tracheal or bronchial rings based on $\mathrm{CT}$; this information still requires bronchoscopy.

Diagnostic imaging of tracheal and bronchial obstructions by magnetic resonance imaging (MRI) has improved substantially over the past few years $[15,16]$. Furthermore, information on cardiovascular malformations can be obtained by application of gadolinium contrast media [17]. Although acquisition times of this imaging modality have decreased considerably during recent years, MDCT is presently much faster than MRI, with acquisition times of 10-30 s. In the present authors' opinion this is a major advantage in children with severe respiratory distress due to tracheal or bronchial stenoses that overcomes the disadvantages of radiation.

According to the present authors' experience, multidetector computed tomography presently appears to be the most valuable primary diagnostic imaging tool in the evaluation of this complex tracheobronchial anomaly but in the case of stenosis, it requires supplementation by flexible bronchoscopy for verification or exclusion of bronchial rings. Bronchography can be abandoned, as multidetector computed tomography reconstructions deliver virtual bronchographic views. Due to major improvements in the diagnosis and treatment of complex bronchial malformations, an increasing number of these patients will survive into adulthood. Since presentation of bridging bronchus will no longer be restricted to the paediatric age group, adult pulmologists should be aware of the typical features of this rare bronchial malformation.

\section{REFERENCES}

1 Evans JA. Aberrant bronchi and cardiovascular anomalies. Am J Med Genet 1990; 35: 46-54.

2 Laforet EG, Starkey GW, Scheff S. Anomalies of upper lobe bronchial distribution. J Thorac Cardiovasc Surg 1962; 43: 595-606.

3 Huizinga E, Smelt GF. Bronchography. Assen, Van Gorcum, 1949.

4 Wells TR, Gwinn JL, Landing BH, Stanley P. Reconsideration of the anatomy of sling left pulmonary artery: the association of one form with bridging bronchus and imperforate anus. Anatomic and diagnostic aspects. $J$ Pediatr Surg 1988; 23: 892-898.

5 Gonzalez-Crussi F, Padilla LM, Miller JK, Grosfeld JL. Bridging bronchus. A previously undescribed airway anomaly. Am J Dis Child 1976; 130: 1015-1018.

6 Wells TR, Stanley P, Padua EM, Landing BH, Warburton D. Serial section-reconstruction of anomalous tracheobronchial branching patterns from CT scan images: bridging bronchus associated with sling left pulmonary artery. Pediatr Radiol 1990; 20: 444-446.

7 Wells LJ, Boyden EA. The development of the bronchopulmonary segments in human embryos of horizons XVII to XIX. Am J Anat 1954; 95: 163-202. 
8 Doehlemann C, Mantel K, Vogl TJ, et al. Pulmonary sling: morphological findings. Pre- and postoperative course. Eur J Pediatr 1995; 154: 2-14.

9 Freedom RM. Pulmonary artery sling. In: Freedom RM, ed. The Natural and Modified History of Congenital Heart Disease. New York, Futura, Blackwell Publishing, 2004; pp. 135-137.

10 Rishavy TJ, Goretsky MJ, Langenburg SE, Klein MD. Anterior bridging bronchus. Pediatr Pulmonol 2003; 35: 70-72.

11 Medina-Escobedo G, Lopez-Corella E. Sling left pulmonary artery, bridging bronchus, and associated anomalies. Am J Med Genet 1992; 44: 303-306.

12 Starshak RJ, Sty JR, Woods G, Kreitzer FV. Bridging bronchus: a rare airway anomaly. Radiology 1981; 140: 95-96.
13 Ziemer G, Heinemann M, Kaulitz R, Freihorst J Seidenberg J, Wilken M. Pulmonary artery sling with tracheal stenosis: primary one-stage repair in infancy. Ann Thorac Surg 1992; 54: 971-973.

14 Bramson RT, Sherman JM, Blickman JG. Pediatric bronchography performed through the flexible bronchoscope. Eur J Radiol 1993; 16: 158-161.

15 Cloutier MM, Schaeffer DA, Hight D. Congenital cystic adenomatoid malformation. Chest 1993; 103: 761-764.

16 Malmgren N, Laurin S, Lundstrom NR. Pulmonary artery sling. Diagnosis by magnetic resonance imaging. Acta Radiol 1988; 29: 7-9.

17 Lee $\mathrm{KH}$, Yoon CS, Choe $\mathrm{KO}$, et al. Use of imaging for assessing anatomical relationships of tracheobronchial anomalies associated with left pulmonary artery sling. Pediatr Radiol 2001; 31: 269-278. 